\title{
EDITORIAL
}

\section{Ventilator-associated pneumonia: approaching the horizon}

\author{
S. Ewig*, E. Müller" and E. Hecker
}

V entilator-associated pneumonia (VAP) continues to be a disturbing problem in the care of intensive care unit (ICU) patients. Although much progress has been achieved in the past two decades, many issues remain unresolved, and mortality still reaches $30-40 \%$ [1, 2]. In this issue of the European Respiratory Journal, two contributions are devoted to VAP. Although quite divergent in scope, they all reflect the many difficulties researchers must face when approaching this issue. After all, it seems that tremendous efforts have to be made to even recognise how such difficulties could be avoided in future, more sophisticated study designs.

The first contribution approaches the question of whether prone positioning reduces the incidence of VAP and its mortality [3]. The authors investigated the effect of prone positioning on the incidence of VAP in a large cohort of 2,409 patients admitted over a $9-y r$ period to 12 French ICUs. They found that prone positioning had no effect on the risk of VAP. Also, they were unable to detect a decrease in mortality, although in patients receiving prone positioning for more than 1 day, mortality may have in fact been reduced. However, mortality was not reduced in patients with acute respiratory distress syndrome (ARDS) at admission.

Positioning as a means to decrease the risk of VAP is continuously attracting much interest. In the 1990s, semirecumbent positioning was shown to reduce VAP rates $[4,5]$. This was explained by a reduction of aspiration of contaminated gastric juice $[4,6]$. Since then, it has become the standard of care in most instances; however, its application in clinical practice continued to be difficult [7]. Prone positioning was primarily introduced as a measure to improve oxygenation. It may, however, also have use as a preventive tool based on better secretion drainage if used early after initiation of mechanical ventilation. Conflicting results were reported as regards the development of VAP [8, 9]. In theory, prone positioning may even favour the development of VAP by increasing the dissemination of pathogens colonising the tracheobronchial tree. However, in a recent meta-analysis comprising four randomised controlled trials studying the

\footnotetext{
*Thoraxzentrum Ruhrgebiet, Kliniken für Pneumologie und Infektiologie, EVK Herne und AugustaKranken-Anstalt Bochum. ${ }^{\#}$ Thoraxzentrum Ruhrgebiet, Klinik für Anästhesiologie, Intensiv- und Notfallmedizin, EVK Herne. "Thoraxzentrum Ruhrgebiet, Klinik für Thoraxchirurgie, EVK Herne, Bochum, Germany.

CORRESPONDENCE: S. Ewig, Thoraxzentrum Ruhrgebiet, Kliniken für Pneumologie und Infektiologie, EVK Herne und Augusta-Kranken-Anstalt Bochum, Bergstrasse 26, 44791 Bochum, Germany. E-mail: ewig@augusta-bochum.de
}

prone position with a total of 1,018 patients, the comparison of prone versus supine position group showed a moderate trend toward better outcomes regarding the incidence of clinically diagnosed VAP among patients in the prone position (OR 0.80, 95\% CI 0.60-1.08) [10].

Several limitations of the study by Mounier et al. [3] must be taken into account. Use of prone positioning was not standardised and may thereby have included selection bias. The median duration of use was 1 day, and no patient received it for longer than 3 days. However, there was also no reduction in risk of VAP in patients receiving prone positioning for at least 2 days. Nevertheless, perhaps the most important message of the study is that it does not favour VAP. Instead, the standard of care was semi-recumbent positioning, thought to be protective against the development of VAP, and prone positioning turned out not to be inferior to semi-recumbent positioning.

However, recent data published by BASSI et al. [11] seriously challenge semi-recumbent positioning. In an animal study, they could show that, following tracheal intubation, gravitational force influences tracheal mucus clearance. When the trachea is oriented above horizontal, a flow of mucus from the proximal trachea toward the lungs is highly associated with bacterial colonisation of the airways and pneumonia. According to these data, optimal positioning could be below horizontal [11]. Whether this will be practical, and if so to what extent, remains to be investigated. In any case, what the authors did was to study prone positioning below horizontal. Thus, further investigation will have to focus on the feasibility of such positioning, possible modifications (e.g. lateral positioning) and its effects on oxygenation and development of VAP.

The second contribution deals with biomarkers in VAP. Are biomarkers useful tools for the prediction of survival and septic shock in patients with VAP? HILLAS et al. [12] performed an observational study of 45 patients with microbiologically confirmed VAP. They measured C-reactive protein (CRP) and procalcitonin (PCT) at days 1, 4 and 7 and evaluated the predictive potential of defined thresholds, as well as kinetics. They found that neither CRP and PCT thresholds nor their kinetics are good predictors of both outcomes, either due to their lack of strength or to the lack of early responsiveness which could influence antimicrobial treatment decisions. There was, however, some potential for PCT on day 1 to predict the development of septic shock.

The results are in contrast to three recent reports. First, PóvOA et al. [13] described that CRP thresholds in serial determinations 
are a useful tool to identify poor outcome from day 4 onwards. Secondly, LUYT et al. [14] identified a strong predictive potential for PCT level on days 1, 3 and 7 for the composite endpoint survival, recurrence and extrapulmonary infection. Finally, SELIGMAN et al. [15] found CRP and PCT kinetics (decreasing values during follow-up) predictive of survival.

These conflicting results highlight the difficulties in evaluating the prognostic potential of biomarkers in patients with VAP. In the study by HiLLAS et al. [12], the number of patients is limited. The microbial spectrum is highly unusual, with excess incidences of (multi)resistant Acinetobacter spp. and Pseudomonas aeruginosa. Accordingly, mortality rates were high (35.6\%). Since biomarkers reflect present and ongoing systemic inflammation, any evaluation of their prognostic potential is highly dependent on the adequacy of, and response to, antimicrobial treatment. Unfortunately, no such information is provided in the report. Conversely, medical and surgical patients are mixed, and ARDS from different causes is included. The real challenge in evaluating biomarkers is to build up a homogeneous study population as far as possible, to carefully define the rate of appropriate initial antimicrobial treatment, to provide the rate of treatment failures, and to assess possible additional confounders of systemic inflammation, such as relapses and superinfections [16]. A good reason to believe that there is a considerable prognostic potential for biomarkers in patients with VAP is provided by studies of patients with lower respiratory tract infections and community-acquired pneumonia (CAP), a much more homogeneous population as regard to host response, expected pathogen patterns and treatment failure rates [17-19]. Therefore, the aforementioned efforts may be rewarding in the future.

Are biomarkers helpful in reducing antibiotic exposure of patients with VAP? This question was addressed in the December issue of the European Respiratory Journal by STOLZ et al. [20], who reported a multicentre randomised controlled trial assigning patients to either a PCT-guided group of antimicrobial treatment discontinuation or to a control group following an antimicrobial discontinuation strategy according to American Thoracic Society/Infectious Diseases Society of America guidelines. The final decision about treatment selection and duration was made by the attending physician. The authors found that the number of antibiotic free days alive 28 days after VAP onset was significantly shorter in the PCT-guided group ( 9.5 days versus 13 days), translating into a reduction in the overall duration of antibiotic therapy of $27 \%$ in the PCT-guided group.

Reduction of antimicrobial exposure may be achieved at several steps of clinical evolution. First, antimicrobial treatment may be stopped $48-72 \mathrm{~h}$ after careful clinical and microbiological re-evaluation of the patient, based on the judgment that the presence of VAP turned out to be unlikely [21-23]. Secondly, de-escalation may be performed, adjusting antimicrobial treatment to culture results. This may result in reduction of dual therapy to monotherapy [24]. Finally, antimicrobial treatment may be stopped at day 8, according to evidence provided by a landmark study [25]. Except for pneumonia caused by bacteremic Staphylococcus aureus and perhaps $P$. aeruginosa, this approach seems adequate for most patients, unless infections other than VAP or complications such as lung abscesses have emerged.
Unfortunately, STOLz et al. [20] focused primarily on the third option, i.e. reduction of overall antimicrobial treatment duration. Obviously, despite the fact that $27 \%$ of patients did not have positive culture results, antimicrobial treatment was not stopped in any of them. Another chance to reduce antimicrobial treatment was missed by the failure to rely on quantitative cultures. Several isolates may not have been "causative microorganisms", and antimicrobial treatment may have been inadequate. De-escalation, although reported to have been taught and applied, was not systematically performed in the control group. Conversely, overall treatment duration was far too long in both groups, since it was well above the 8 days established as appropriate length of stay [25]. Although treatment discontinuation at day 7 was significantly higher in the PCT-guided group (35\% versus 18\%) and although, unfortunately, antimicrobial treatment for reasons other than VAP was not subtracted from total exposure duration, the value of a biomarker-based strategy is highly questionable if it is reported to fall behind an established clinical target. A similar failure also devalued the conclusions from a trial of PCT-guided treatment duration in patients with CAP [26]. The clinical target is not to blame if clinicians are not willing or able to adhere with it. Instead, continuous medical teaching seems mandatory, insisting on the many options to reduce antimicrobial exposure.

The study by STOLz et al. [20] would have been better designed as an interventional study, clearly defining and executing treatment stops, de-escalation and duration, and providing options for overruling in individual cases. As it stands, PCTguided strategy primarily seems to be an educational rather than physiological tool of reduction of antimicrobial treatment exposure. The critical issue, however, is whether biomarkers such as PCT allow the cessation of antimicrobial treatment, an increase in de-escalation rates, and a reduction of antimicrobial treatment duration beyond the clinical target of 8 days. There is evidence from a small Swiss study that a protocol based on serial PCT measurement allows reduction of antibiotic treatment duration and exposure in patients with severe sepsis and septic shock, without apparent harm, to a median treatment duration of even 6 days. This was true despite the option to overrule recommendations by PCT values [27]. Nevertheless, so far, this issue remains to be confirmed and should urgently be investigated further.

In conclusion, compared with lower respiratory tract infections and $\mathrm{CAP}$, current data regarding the appropriate use of biomarkers in VAP are less conclusive. CRP may be used primarily used to confirm the appropriateness of antimicrobial treatment [28]. PCT holds much promise as a tool to reduce antimicrobial exposure; however, the cost-effectiveness of PCT kinetics has not been established. After all, valuable progress is also to know where to go on from here.

\section{STATEMENT OF INTEREST}

A statement of interest for S. Ewig can be found at www.erj. ersjournals.com/misc/statements.dtl

\section{REFERENCES}

1 Torres A, Ewig S, Lode H, et al. Defining, treating and preventing hospital acquired pneumonia: European perspective. Intensive Care Med 2009; 35: 9-29. 
2 Torres A, Ewig S, Lode $\mathrm{H}$, et al. Hospital-acquired pneumonia in Europe. Eur Respir J 2009; 33: 951-952.

3 Mounier R, Adrie C, Français A, et al. Study of prone positioning to reduce ventilator-associated pneumonia in hypoxaemic patients. Eur Respir J 2010; 35: 795-804.

4 Torres A, Serra-Batlles J, Ros E, et al. Pulmonary aspiration of gastric contents in patients receiving mechanical ventilation: the effect of body position. Ann Intern Med 1992; 116: 540-543.

5 Drakulovic MB, Torres A, Bauer TT, et al. Supine body position as a risk factor for nosocomial pneumonia in mechanically ventilated patients: a randomised trial. Lancet 1999; 354: 1851-1858.

6 Orozco-Levi M, Torres A, Ferrer M, et al. Semirecumbent position protects from pulmonary aspiration but not completely from gastroesophageal reflux in mechanically ventilated patients. Am J Respir Crit Care Med 1995; 152: 1387-1390.

7 van Nieuwenhoven CA, Vandenbroucke-Grauls C, van Tiel FH, et al. Feasibility and effects of the semirecumbent position to prevent ventilator-associated pneumonia: a randomized study. Crit Care Med 2006; 34: 396-402.

8 Mancebo J, Fernandez R, Blanch L, et al. A multicenter trial of prolonged prone ventilation in severe acute respiratory distress syndrome. Am J Respir Crit Care Med 2006; 173: 1233-1239.

9 Guerin C, Gaillard S, Lemasson S, et al. Effects of systematic prone positioning in hypoxemic acute respiratory failure: a randomized controlled trial. JAMA 2004; 292: 2379-2387.

10 Alexiou VG, Ierodiakonou V, Dimopoulos G, et al. Impact of patient position on the incidence of ventilator-associated pneumonia: a meta-analysis of randomized controlled trials. J Crit Care 2009; 24: 515-522.

11 Bassi GL, Zanella A, Cressoni M, et al. Following tracheal intubation, mucus flow is reversed in the semirecumbent position: possible role in the pathogenesis of ventilator-associated pneumonia. Crit Care Med 2008; 36: 518-525.

12 Hillas G, Vassilakopoulos T, Plantza P, et al. C-reactive protein and procalcitonin as predictors of survival and septic shock in ventilator-associated pneumonia. Eur Respir J 2010; 35: 805-811.

13 Póvoa P, Coelho L, Almeida E, et al. Early identification of intensive care unit-acquired infections with daily monitoring of Creactive protein: a prospective observational study. Crit Care 2006; 10: R63.

14 Luyt CE, Guérin V, Combes A, et al. Procalcitonin kinetics as a prognostic marker of ventilator-associated pneumonia. Am J Respir Crit Care Med 2005; 171: 48-53.
15 Seligman R, Meisne M, Lisboa TC, et al. Decreases in procalcitonin and C-reactive protein are strong predictors of survival in ventilator-associated pneumonia. Crit Care 2006; 10: R125.

16 Ioanas M, Ewig S, Torres A. Treatment failures in patients with ventilator-associated pneumonia. Infect Dis Clin North Am 2003; 17: 753-771.

17 Christ-Crain M, Jaccard-Stolz D, Bingisser R, et al. Effect of procalcitonin-guided treatment on antibiotic use and outcome in lower respiratory tract infections: cluster-randomised, singleblinded intervention trial. Lancet 2004; 363: 600-607.

18 Stolz D, Christ-Crain M, Bingisser R, et al. Antibiotic treatment of exacerbations of COPD: a randomized, controlled trial comparing procalcitonin-guidance with standard therapy. Chest 2007; 131: 9-19.

19 Stolz D, Christ-Crain M, Morgenthaler NG, et al. Copeptin, Creactive protein, and procalcitonin as prognostic biomarkers in acute exacerbation of COPD. Chest 2007; 131: 1058-1067.

20 Stolz D, Smyrnios N, Eggimann P, et al. Procalcitonin for reduced antibiotic exposure in ventilator associated pneumonia: a randomised study. Eur Respir J 2009; 34: 1364-1375.

21 Fagon JY, Chastre J, Wolff M, et al. Invasive and noninvasive strategies for management of suspected ventilator-associated pneumonia. A randomized trial. Ann Intern Med 2000; 132: 621-630.

22 Singh N, Rogers $\mathrm{P}$, Atwood CW, et al. Short-course empiric antibiotic therapy for patients with pulmonary infiltrates in the intensive care unit. A proposed solution for indiscriminate antibiotic prescription. Am J Respir Crit Care Med 2000; 162: 505-511.

23 Torres A, Ewig S. Diagnosing ventilator-associated pneumonia. N Engl J Med 2004; 350: 433-435.

24 Niederman MS. The importance of de-escalating antimicrobial therapy in patients with ventilator-associated pneumonia. Semin Respir Crit Care Med 2006; 27: 45-50.

25 Chastre J, Wolff M, Fagon JY, et al. Comparison of 8 vs 15 days of antibiotic therapy for ventilator-associated pneumonia in adults: a randomized trial. JAMA 2003; 290: 2588-2598.

26 Christ-Crain M, Stolz D, Bingisser R, et al. Procalcitonin guidance of antibiotic therapy in community-acquired pneumonia: a randomized trial. Am J Respir Crit Care Med 2006; 174: 84-93.

27 Nobre V, Harbarth S, Graf JD, et al. Use of procalcitonin to shorten antibiotic treatment duration in septic patients: a randomized trial. Am J Respir Crit Care Med 2008; 177: 498-505.

28 Lisboa T, Seligman R, Diaz E, et al. C-reactive protein correlates with bacterial load and appropriate antibiotic therapy in suspected ventilator-associated pneumonia. Crit Care Med 2008; 36: 166-171. 